\title{
Principales características de la investigación biomédica actual, en Chile
}

Miembros de Número, Academia de Medicina del Instituto de Chile. ${ }^{2}$ Presidente de la Academia. ${ }^{3}$ Facultad de Medicina, Pontificia Universidad Católica de Chile. ${ }^{4}$ Facultad de Medicina, Universidad de Chile, Campus Occidente. ${ }^{5}$ Facultad de Medicina, Universidad de Chile, Campus Oriente. Santiago de Chile.

Estudio encomendado por la Academia de Medicina, con motivo de la XIX Reunión del Consejo Directivo de la Asociación de Academias de Medicina de América Latina, España y Portugal (ALANAM), realizada en Santiago de Chile, 28-30 de octubre de 2010.

Correspondencia a: Dra. Gloria Valdés S.,

Centro de Investigaciones Médicas, Escuela de Medicina Pontificia Universidad Católica de Chile. Marcoleta 391. Teléfono 3548176 Fax 6321924 E-mailgvaldes@med.puc.cl

\author{
GLORIA VALDÉS S. ${ }^{1,3}$, RODOLFO ARMAS M. ${ }^{1,2,4}$, HUMBERTO REYES B. ${ }^{1,5}$
}

\section{Main characteristics of current biomedical research, in Chile}

Background: Biomedical research is a fundamental tool for the development of a country, requiring human and financial resources. Aim: To define some current characteristics of biomedical research, in Chile. Methods: Data on entities funding biomedical research, participant institutions, and the number of active investigators for the period 2007-2009 were obtained from institutional sources; publications indexed in PubMed for 2008-2009 were analysed. Results: Most financial resources invested in biomedical research projects (approximately US\$ 19 million per year) came from the "Comisión Nacional de Investigación Científica y Tecnológica" (CONICYT), a state institution with 3 independent Funds administering competitive grant applications open annually to institutional or independent investigators in Chile. Other sources and universities raised the total amount to US\$ 26 million. Since 2007 to 2009, 408 investigators participated in projects funded by CONICYT. The main participant institutions were Universidad de Chile and Pontificia Universidad Católica de Chile, both adding up to 84\% of all funded projects. Independently, in 2009, 160 research projects -mainly multi centric clinical trials-received approximately US\$24 million from foreign pharmaceutical companies. Publications listed in PubMed were classified as "clinical research" ( $n=879$, including public health) or "basic biomedical research" $(n=312)$. Conclusions: Biomedical research in Chile is mainly supported by state funds and university resources, but clinical trials also obtained an almost equivalent amount from foreign resources. Investigators are predominantly located in two universities. A small number of $M D-P h D$ programs are aimed to train and incorporate new scientists. Only a few new Medical Schools participate in biomedical research. A National Registry of biomedical research projects, including the clinical trials, is required among other initiatives to stimulate research in biomedical sciences in Chile.

(Rev Med Chile 2012; 140: 484-492).

Key words: Biomedical research; Chile; Clinical trials as topic.
I nvestigación biomédica es la investigación científica destinada a obtener conocimientos y proponer soluciones a problemas de salud de las personas y la población. En una visión amplia, incluye investigación en ciencias biológicas, orientada a la fisiología humana (investigación biomédica básica); en medicina clínica, para prevenir, diagnosticar, tratar, determinar la severidad o la prevalencia de una enfermedad; en salud pública, enfocada a problemas poblacio- nales; en administración en salud, para mejorar la gestión y eficiencia de la acción médica; y en ciencias sociales, para reconocer comportamientos individuales y colectivos que puedan influir sobre la salud.

Los objetivos de la investigación biomédica son:

1. Satisfacer la necesidad del ser humano de incrementar sus conocimientos, para entender y mejorar su propia existencia. 
2. Contar con investigadores que enseñen a los estudiantes los fundamentos de las ciencias biológicas, psicológicas y sociales, en un marco de disciplina y rigurosidad científica. Ello atrae nuevas vocaciones al quehacer científico y familiariza a los estudiantes para analizar críticamente el conocimiento nuevo que, durante su ejercicio profesional, modificará al recibido en la etapa de formación universitaria.

3. Incorporar al trabajo clínico conocimientos generados en las disciplinas básicas y preclínicas.

4. Descubrir recursos diagnósticos o terapéuticos, o elegir la mejor forma de usar los ya conocidos.

5. Identificar problemas sanitarios de la población, aprender a corregirlos y prevenirlos.

6. Elevar la capacidad de resolver problemas complejos y abordar factores sociales determinantes de la salud ${ }^{1-5}$.

El presente estudio pretende actualizar características importantes de la investigación biomédica en Chile: los recursos disponibles (financieros y humanos) y sus resultados, reflejados en publicaciones en revistas "de corriente principal".

\section{Material y Método}

Como índices de la situación contemporánea se recolectaron datos del trienio 2007-2009.

\section{Financiamiento de la investigación biomédica}

La información sobre recursos financieros aportados por el estado para investigación biomédica, se obtuvo de la Comisión Nacional de Investigación Científica y Tecnológica (CONICYT), que depende del Ministerio de Educación. Se identificaron los proyectos de investigación financiados por su "Fondo de Investigación Científica y Tecnológica” (FONDECYT), el "Fondo de Fomento al Desarrollo Científico y Tecnológico" (FONDEF), y el "Fondo Nacional de Investigación y Desarrollo en Salud” (FONIS, cuya organización y financiamiento comparten CONICYT y el Ministerio de Salud).

CONICYT selecciona los proyectos a través de concursos anuales, competitivos, abiertos a investigadores de instituciones públicas o privadas, estableciendo estándares de calidad. Sus tres Fondos tienen manejo independiente. Aproximadamente $75 \%$ de los proyectos financiados por FONDECYT en su “área de Medicina”, corresponden a investigación biomédica. FONDEF reparte en igual proporción monetaria su apoyo a proyectos de salud pública y clínicos, exigiendo aportes de la entidad beneficiada y de empresas que contribuyen al proyecto. FONIS ofrece recursos para investigaciones aplicadas que contribuyan a mejorar las políticas públicas sanitarias; aproximadamente $66 \%$ de los proyectos que financia son de salud pública.

En CONICYT y en los Ministerios de Salud y de Educación se obtuvo información sobre otros organismos estatales que aportan recursos.

Además, una encuesta dirigida en el año 2010 a los Encargados de Investigación de universidades nacionales que imparten la carrera de Medicina, solicitó información sobre recursos internos o externos, no estatales, otorgados a proyectos de investigación biomédica.

\section{Número de investigadores activos}

Para un recuento de los investigadores nacionales y su filiación institucional, se identificaron el investigador principal y los co-investigadores en proyectos seleccionados y administrados por CONICYT. En la nómina resultante se eliminó la duplicidad de menciones de participantes en proyectos simultáneos.

Se estableció como requisito que los investigadores hubieran diseñado y realizado el proyecto de investigación, con independencia de la entidad que lo financió.

\section{Formación de recursos humanos}

Se eligió como índice, la disponibilidad de programas de Doctorado en Ciencias Médicas. Los datos se obtuvieron de las universidades que cuentan con estos Programas.

\section{Publicaciones científicas}

Dentro de la investigación biomédica identificamos como investigación clínica aquella que cumplía los criterios propuestos en 1979 por Robert y Suzanne Fletcher. Dichos autores propusieron calificar como "investigación clínica" no sólo los estudios epidemiológicos y de seguimiento clínico que cumplen estrictamente los criterios de la investigación científica, sino también presentaciones de casos, ensayos clínicos no controlados, estudios retrospectivos y revisiones de temas, porque son actividades de investigación propias de la medicina que prestan una utilidad evidente 
para la práctica clínica ${ }^{6}$. Un tipo particular de investigación clínica son los ensayos clínicos ("clinical trials") $)^{7,8}$.

Las publicaciones biomédicas chilenas, en los años 2008 y 2009, fueron extraídas de la base de datos MEDLINE/PubMed, utilizando como "buscadores" los términos en español o inglés explicitados en Anexo, disponible por solicitud a la autora corresponsal.

Se clasificó "investigación biomédica básica" a publicaciones sobre experimentación en animales, estudios en tejidos, células o fracciones celulares, destinada al conocimiento de la fisiología humana y orientada por problemas clínicos. Se clasificó "investigación clínica" a los estudios en seres humanos, enfocados en problemas clínicos ${ }^{1}$, incluyendo publicaciones referidas a salud pública y administración.

Entre los estudios colaborativos internacionales, se incluyeron únicamente los que reconocieron entre sus autores la participación de chilenos con co-patrocinio de una institución nacional.

Dos autores del presente trabajo clasificaron independientemente cada publicación como "investigación biomédica básica" o "investigación clínica", excluyendo del análisis posterior a las que consideraron fuera del ámbito biomédico ya definido.

Resultados

Financiamiento de la investigación biomédica

La Tabla 1 muestra el número de Proyectos financiados por FONDECYT, en su área "Medicina”, en el trienio 2007-2009, según la Unidad o institución en que se realizaron. La mayor proporción de proyectos $(84,2 \%)$ procedieron de Facultades o Institutos de la Universidad de Chile o la Pontificia Universidad Católica de Chile.
En sus tres primeros años, FONIS financió 83 proyectos. La Tabla 2 los clasificó en 4 áreas temáticas, y muestra que la mayoría se enfocaron en temas de Salud Pública o Medicina Clínica.

Tabla 1. Proyectos FONDECYT del Área de Medicina 2007-2009, según entidad investigadora

\begin{tabular}{|c|c|c|}
\hline & (n) & $\begin{array}{l}\% \text { del } \\
\text { total }\end{array}$ \\
\hline Universidad de Chile & 59 & 54,6 \\
\hline Facultad de Medicina & 29 & \\
\hline Hospital Clínico & 6 & \\
\hline INTA & 15 & \\
\hline Facultad de Ciencias & 1 & \\
\hline $\begin{array}{l}\text { Facultad de Ciencias Químicas y } \\
\text { Farmacéuticas }\end{array}$ & 5 & \\
\hline Facultad de Odontología & 3 & \\
\hline Pontificia Universidad Católica de Chile & 32 & 29,6 \\
\hline Facultad de Medicina & 26 & \\
\hline Facultad de Ciencias Biológicas & 5 & \\
\hline Facultad de Ciencias Sociales & 1 & \\
\hline Universidad Austral de Chile & 4 & 3,7 \\
\hline Universidad de Concepción & 4 & 3,7 \\
\hline Facultad de Medicina & 1 & \\
\hline Facultad de Ciencias Biológicas & 1 & \\
\hline Facultad de Medicina Veterinaria & 1 & \\
\hline Facultad de Odontología & 1 & \\
\hline Universidad de Santiago de Chile & 2 & 1,8 \\
\hline Departamento de Informática & 1 & \\
\hline $\begin{array}{l}\text { Facultad de Ciencias Químicas y } \\
\text { Biología }\end{array}$ & 1 & \\
\hline Otras Instituciones* & 7 & 6,5 \\
\hline Total & 205 & 100 \\
\hline
\end{tabular}

* Uno de cada una de las siguientes instituciones: Universidades de Los Andes, de Tarapacá, de la Frontera, Católica del Maule, Federico Santa María; Grupo BIOS y Clínica Las Condes.

Tabla 2. Proyectos aprobados por FONIS, según disciplina

\begin{tabular}{|lcccccccc|}
\hline $\begin{array}{l}\text { Concurso } \\
\text { (año) }\end{array}$ & $\begin{array}{c}\text { Total } \\
\text { (n) }\end{array}$ & $\begin{array}{c}\text { Clínicos } \\
\text { Epidemiología } \\
\text { clínica (n) }\end{array}$ & $\begin{array}{c}\text { Otros } \\
\text { (n) }\end{array}$ & $\begin{array}{c}\text { Total } \\
\text { (n) }\end{array}$ & $\begin{array}{c}\text { Programas } \\
\text { Salud Pública } \\
\text { (n) }\end{array}$ & $\begin{array}{c}\text { Economía de } \\
\text { Salud } \\
\text { (n) }\end{array}$ & $\begin{array}{c}\text { Gestión de } \\
\text { Servicio } \\
\text { (n) }\end{array}$ \\
\hline $1^{\circ}$ & 25 & 1 & 4 & 5 & 18 & 1 & 1 \\
\hline $2^{\circ}$ & 31 & 4 & 4 & 8 & 19 & & 4 \\
\hline $3^{\circ}$ & 27 & 2 & 11 & 13 & 7 & 1 & 6 \\
\hline Total & 83 & 7 & 19 & 26 & 44 & 2 & 11 \\
\hline
\end{tabular}


La Tabla 3 muestra los montos asignados a proyectos del área de Medicina, durante el año 2009, por cada Fondo dependiente de CONICYT, más los recursos de otras fuentes, administrados por esta institución.

La Figura 1 muestra los aportes internos o externos, independientes de CONICYT, que destinaron las Facultades de Medicina para investigación biomédica. Resalta la gran proporción de recursos financieros invertidos por la Pontificia Universidad Católica de Chile y la Universidad de Chile.

Tabla 3. Fondos aportados por y a través de CONICYT para Proyectos de Investigación. Nuevos y de Renovación del Área de la Salud en miles de pesos, en 2009

\begin{tabular}{|c|c|c|c|c|}
\hline Fondo & Concurso & Aporte de CONICYT & Otros aportes & Total (en M\$) \\
\hline FONDECYT $^{(a)}$ & $\begin{array}{l}\text { Regular } \\
\text { Post Doctorado } \\
\text { Iniciación en Investigación } \\
\text { Incentivo Cooperac Internac. } \\
\text { Total FONDECYT }\end{array}$ & $\begin{array}{r}5.613 .138 \\
39.832 \\
736.142 \\
6.100\end{array}$ & & 6.395 .212 \\
\hline FONDEF (b) & $\begin{array}{l}\text { CONICYT } \\
\text { Unidad Beneficiaria } \\
\text { Empresas y entidades asociadas } \\
\text { Total FONDEF y aportes agregados }\end{array}$ & 1.261 .771 & $\begin{array}{l}489.989 \\
481.801\end{array}$ & 2.233 .561 \\
\hline FONIS (c) & & 250.000 & $250.000^{(d)}$ & 500.000 \\
\hline Total Progran & de CONICYT & 7.906 .983 & 1.221 .790 & 9.128 .773 \\
\hline
\end{tabular}

(a)FONDECYT: Fondo Nacional de Desarrollo Científico y Tecnológico; (b)FONDEF: Fondo de Fomento al Desarrollo Científico y Tecnológico; (c)FONIS: Fondo Nacional de Investigación y Desarrollo en Salud; (d)Aporte del Ministerio de Salud.

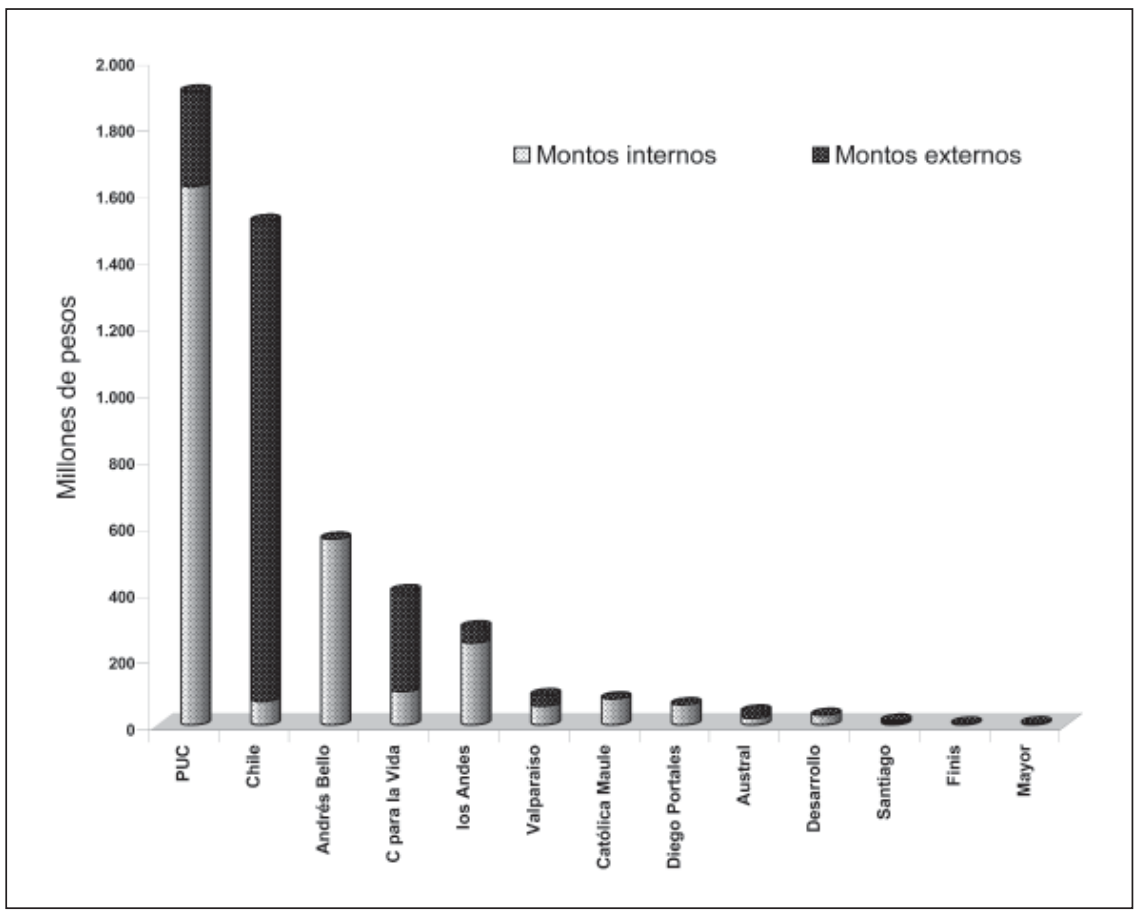

Figura 1. Recursos anuales dedicados por las universidades, y por la Fundación para la Vida, calculados según datos proporcionados para el período 2008-2009. 
Número de investigadores activos

En el trienio 2007-2009, 408 personas calificaron como investigadores activos en biomedicina, participantes en proyectos financiados por CONICYT.

Este número es 19\% menor al contabilizado en el año 2005 por la Academia Chilena de Ciencias: 504 investigadores, cifra obtenida en encuestas a investigadores registrados en CONICYT y las universidades, a sociedades científicas y médicas. Este criterio fue más amplio que el empleado en el presente estudio. Los investigadores en el área biomédica representaron el mayor número por disciplina, en el país (Tabla 4, con datos reproducidos de la referencia 9).

Ubicación institucional de los investigadores

En los proyectos biomédicos financiados por FONDECYT, la mayoría de los investigadores son académicos de la Universidad de Chile y de la Pontificia Universidad Católica de Chile, donde se realizaron $84 \%$ de estos proyectos (Tabla 1).

Formación de nuevos investigadores

La Tabla 5 muestra el número de alumnos que ingresaron a Programas de Doctorado en Ciencias Médicas, en universidades chilenas, desde 1995. Dirigidos a formar investigadores para el área biomédica, reciben actualmente 8 a 10 alumnos por año. El Programa de la Universidad de Chile está dirigido a médicos que, junto al Doctorado, cursan un programa de especialidad médica. Los de la Pontificia Universidad Católica de Chile y de la Universidad Austral de Chile están abiertos a médicos y a egresados de disciplinas afines. La Universidad de La Frontera admite graduados de Medicina u Odontología, con título de especialistas, o de Magíster en investigación clínica o equivalente.
Tabla 4. Investigadores en distintas disciplinas, cuantificados por catastro incluido en "Análisis y proyecciones de la Ciencia Chilena 2005"9

\begin{tabular}{|lcc|}
\hline Disciplina & $\begin{array}{c}\text { Investigadores } \\
\text { (n) }\end{array}$ & \% del total \\
\hline Biomedicina & 504 & 18,86 \\
\hline Biología & 424 & 15,77 \\
\hline Ciencias Químicas & 390 & 14,45 \\
\hline Ciencias de la Ingeniería & 254 & 9,41 \\
\hline Ciencias Agronómicas y Forestales & 206 & 7,63 \\
\hline Física & 205 & 7,59 \\
\hline Ciencias Ambientales & 170 & 6,30 \\
Ciencias Matemáticas & 169 & 6,26 \\
Ciencias del Mar & 157 & 5,81 \\
Ciencias de la Tierra & 155 & 5,74 \\
Astronomía & 64 & 2,37 \\
Total & 2.698 & 100 \\
\hline
\end{tabular}

El Directorio de Investigadores en Biomedicina se confeccionó mediante cuestionario electrónico enviado a 1.600 personas seleccionadas entre los investigadores responsables y co-investigadores de FONDECYT, FONDEF en el área de Biología y Medicina, y de FONIS, entre el 2000 y el 2005, y los miembros de las Sociedades Científicas y Médicas de Chile.

Tabla 5. Alumnos ingresados a los Programas de Doctorado en Ciencias Médicas entre los años 1995 y 2011, en las diferentes instituciones que los imparten

\begin{tabular}{|c|c|c|c|c|c|}
\hline & $\begin{array}{l}\text { PUC(1) } \\
\text { (n) }\end{array}$ & $\begin{array}{c}\mathbf{U} \text { de } \mathbf{C H}^{(2)} \\
\text { (n) }\end{array}$ & $\begin{array}{l}\text { UFRO }^{(3)} \\
\text { (n) }\end{array}$ & $\begin{array}{l}\text { U Austral de } \\
\text { Chile (n) }\end{array}$ & $\begin{array}{l}\text { Total } \\
\text { (n) }\end{array}$ \\
\hline 1995 & 2 & & & & 2 \\
\hline 1996 & 1 & & & & 1 \\
\hline 1997 & 2 & & & & 2 \\
\hline 1998 & 3 & & & & 3 \\
\hline 1999 & 1 & & & & 1 \\
\hline 2000 & 1 & 2 & & & 3 \\
\hline 2001 & 3 & 3 & & & 6 \\
\hline 2002 & 4 & 3 & & & 7 \\
\hline 2003 & 6 & 4 & & & 10 \\
\hline 2004 & 3 & 4 & & & 7 \\
\hline 2005 & 6 & 5 & & & 11 \\
\hline 2006 & 3 & 4 & & & 7 \\
\hline 2007 & 3 & 3 & & & 6 \\
\hline 2008 & 4 & 3 & 3 & & 10 \\
\hline 2009 & 4 & 3 & 3 & & 10 \\
\hline 2010 & 3 & 2 & 3 & & 8 \\
\hline 2011 & 3 & 3 & 5 & 5 & 16 \\
\hline
\end{tabular}

(1) Pontificia Universidad Católica de Chile; (2)Universidad de Chile; (3)Universidad de la Frontera. 


\section{Publicaciones científicas}

En la base de datos MEDLINE/PubMed (2007-2009) se identificaron 1.729 publicaciones generadas por autores chilenos, trabajando en el país. Se excluyeron del análisis 538 títulos que no cumplían las características de una investigación biomédica.

Entre las 1.191 publicaciones que cumplieron los criterios de inclusión, 879 (73,8\%) fueron clasificadas como "investigación clínica" y 312 (26,2\%) como "investigación biomédica básica" (Tabla 6). El análisis individual, por dos autores del presente estudio, tuvo concordancia de 93,7\% al asignar las publicaciones a una u otra área, o eliminarlas del estudio; una revisión posterior, en conjunto, permitió consensuar las divergencias. Sólo 7 publicaciones se calificaron como "ensayos clínicos": 3 de la Universidad de Chile, 2 de la

Tabla 6. Publicaciones biomédicas realizadas en instituciones chilenas e indexadas en PubMed, en el período 2008-2009

\begin{tabular}{|c|c|c|c|c|}
\hline Institución & Médicas & Biomédicas & Total & $\%$ \\
\hline \multicolumn{5}{|l|}{ Universidad de Chile } \\
\hline Facultad Medicina & 357 & 133 & 490 & \\
\hline Otras Facultades* & 21 & 6 & 27 & \\
\hline Total & & & & 43,4 \\
\hline \multicolumn{5}{|l|}{ Universidad Católica } \\
\hline Facultad de Medicina & 211 & 28 & 239 & \\
\hline Otras Facultades** & 7 & 44 & 51 & \\
\hline Total & & & & 24,3 \\
\hline Universidad del Desarrollo/Clínica Alemana & 46 & 12 & 58 & 4,9 \\
\hline Universidad Austral & 22 & 22 & 44 & 3,7 \\
\hline Universidad de la Frontera & 31 & 11 & 42 & 3,5 \\
\hline Clínica Las Condes & 40 & & 40 & 3,3 \\
\hline Universidad de Concepción & 28 & 10 & 38 & 3,2 \\
\hline Universidad de Santiago & 11 & 19 & 30 & 2,5 \\
\hline Universidad de Valparaíso & 13 & 5 & 18 & 1,5 \\
\hline Universidad de Talca & 13 & 5 & 18 & 1,5 \\
\hline Universidad Diego Portales & 16 & & 16 & 1,3 \\
\hline Universidad Católica del Norte & 7 & 3 & 10 & 0,8 \\
\hline Universidad Católica de Valparaíso & 3 & 6 & 9 & 0,7 \\
\hline Universidad del Bío-Bío & 4 & 5 & 9 & 0,7 \\
\hline Universidad Mayor & 8 & 0 & 8 & 0,7 \\
\hline Universidad de los Andes & 7 & 0 & 7 & 0,6 \\
\hline Universidad de Antofagasta & 6 & 1 & 7 & 0,7 \\
\hline Fundación Ciencias para la Vida & 2 & 5 & 7 & 0,6 \\
\hline Universidad Andrés Bello & 5 & 1 & 6 & 0,5 \\
\hline Hospital Militar & 6 & & 6 & 0,5 \\
\hline Hospital Naval & 6 & & 6 & 0,5 \\
\hline Otras Instituciones*** & 9 & 1 & 10 & 0,8 \\
\hline Total Publicaciones & 879 & 312 & 1.191 & \\
\hline$\%$ & 73,8 & 26,2 & 100 & 100 \\
\hline
\end{tabular}

*Facultades de Odontología, Ciencias, Química y Farmacia, Bioquímica y Biología Molecular, Ciencias Veterinarias e Ingeniería, Instituto de Dinámica Celular y Biotecnología, Centro de Neurociencias Cognitivas, Centro de Investigación Avanzada en Educación. **Facultad de Ciencias Biológicas. ***con menos de 5 publicaciones (Universidades Católica del Maule, Tarapacá, Santísima Concepción, Clínicas FACH, DIPRECA, Santa María). 
Pontificia Universidad Católica de Chile, 1 de la Universidad del Desarrollo/Clínica Alemana y 1 de la Universidad Austral de Chile.

La mayoría de las publicaciones se generaron en Facultades de Medicina; un pequeño número procedió de Facultades de Odontología, Bioquímica, Farmacia o Enfermería u otros organismos. En 3 publicaciones se reconoció al Ministerio de Salud como una de las instituciones participantes.

Del total de las publicaciones analizadas, $67,2 \%$ pertenecieron a investigadores de la Universidad de Chile o de la Pontificia Universidad Católica de Chile. Dentro de la Universidad de Chile, la mayor proporción perteneció al Instituto de $\mathrm{Nu}-$ trición y Tecnología de Alimentos (INTA). Entre las universidades privadas destacó la Universidad del Desarrollo/Clínica Alemana, que sobrepasó a algunas universidades tradicionales y las demás universidades privadas. Entre las instituciones privadas destacó Clínica Las Condes.

\section{Discusión}

En Chile, el financiamiento estatal de la investigación científica, incluyendo la biomédica, proviene prioritariamente de una institución: CONICYT. Además, financian investigación en diferentes áreas, incluyendo salud, la Iniciativa Científica Milenio, dependiente del Ministerio de Planificación, la Corporación de Fomento de la Producción, dependiente del Ministerio de Economía, y algunas universidades.

Tal como se observó en la década de 1980, los proyectos de investigación biomédica y las publicaciones se concentraron en las mismas dos universidades $^{10-12}$. Este fenómeno ocurre también en otros países de América Latina: en México, Colombia y Perú, menos de 10\% de las universidades producen más de $50 \%$ de las publicaciones biomédicas nacionales en revistas de corriente principal $^{13}$.

Los datos sobre disponibilidad de recursos estatales, sus vías y mecanismos de adjudicación, los investigadores respaldados con estos recursos, y la distribución de los recursos en cada proyecto, son públicamente accesibles. En cambio, los aportes de entidades extranjeras para investigación biomédica en el país, están sólo parcialmente disponibles ${ }^{14,15}$.

La productividad nacional en publicaciones se analizó solamente con aquellas identificadas en MEDLINE/PubMed, de la U.S.National Library of Medicine, considerada la principal base de datos mundial sobre publicaciones científicas, que indexa aproximadamente 5.000 revistas seleccionadas con criterios estrictos que las ubican en la "corriente principal". Otras bases de datos permiten acceder a un número mayor de publicaciones, pero con criterios de selección menos estrictos. Scopus, empresa privada ligada a la Editorial Elsevier, cubre alrededor de 18.000 revistas y es la base de datos utilizada por The SCImago Journal \& Country Rank ${ }^{13}$. Esta última entidad informó que en el bienio 2008-2009 hubo 2.133 "documentos citables" originados por autores chilenos, en el área "Medicina”. En el año 2010, su "ranking internacional", con datos de 211 países, ubicó a Chile en el lugar $43^{\circ}$, con 1.269 "documentos citables", que representaron $6,6 \%$ de las publicaciones latinoamericanas y $0,2 \%$ del total mundial. En Latinoamérica, Chile ocupó el $4^{\circ}$ lugar, precedido por Brasil ( $13^{\circ}$ en el ranking mundial), México (31 $)$ y Argentina (35०). Comparados con los datos de años precedentes, habría un aumento progresivo en las publicaciones de estos 4 países, pero ello pudo estar influido por un aumento en el número de revistas indexadas, más que en la productividad de publicaciones: en el año 2000 Scopus identificó 6.394 "documentos citables" en el mundo, cifra que en 2010 ascendió a 18.932 .

Cabe resaltar que entre las publicaciones indexadas por PubMed en los años 2008 y 2009 se identificaron solamente 7 "ensayos clínicos" hechos en Chile, por autores nacionales. Este número contrasta con la información obtenida sobre la participación de empresas extranjeras, proveedoras de insumos para la medicina, patrocinando "ensayos clínicos" en Chile ${ }^{16,17}$. El registro de los U.S. National Institutes of Health (NIH) reconoció 96.485 ensayos en ejecución al 30 de septiembre de 2010, en 168 países, de los cuales 6.341 (6,6\%) se desarrollaban en 24 países de América Latina y el Caribe. Chile figuró con 569 estudios, ocupando el lugar $41^{\circ}$ en el registro mundial y el $4^{\circ}$ en América Latina, precedido por Brasil, México y Argentina. En nuestro país, como en el resto del mundo, la mayor parte de estos estudios (82\%) correspondían a ensayos de fármacos nuevos y casi todos eran patrocinados por empresas de investigación y desarrollo (I\&D) extra nacionales. 
En 2009, las compañías farmacéuticas afiliadas a la Cámara de la Industria Farmacéutica de Chile habrían invertido aproximadamente 24 millones de dólares para apoyar 160 estudios clínicos, casi todos multicéntricos (con un promedio de 3,3 países para cada estudio $)^{18}$. Esto indica que, en ese momento, los recursos financieros aportados para ensayos clínicos por empresas de I\&D extranjeras semejaban a los recursos disponibles para toda la investigación biomédica realizada en Chile con respaldo estatal, universitario o de otras fuentes nacionales (aproximadamente 26 millones de dólares).

Hay que tener presente que desconocemos los mecanismos empleados por las empresas de I\&D para seleccionar a las personas participantes en los ensayos clínicos que patrocinan, cuánto interviene los participantes nacionales en el diseño del estudio, en la elaboración de los manuscritos generados y si tienen independencia para informar los resultados, particularmente cuando son negativos. En países anglosajones el proceso está más regulado, se califica como "trialist" a las personas que conducen los "clinical trials" y estas investigaciones se inscriben en registros de acceso público libre, informando a las instituciones involucradas en ellas el monto y la distribución de recursos y honorarios que generan, con las correspondientes responsabilidades tributarias y la obligación de declarar eventuales conflictos de intereses cuando participan, además, en otras actividades académicas, institucionales y profesionales.

Es justo reconocer que a través de los ensayos clínicos se pueden generar ingresos institucionales por "overhead" y honorarios para quienes trabajan en ellos, aportes en equipos, entrega de conocimientos nuevos y oportunidades de acceder a medicamentos o tecnologías nuevas, muchas veces de alto costo. Pero, para ser reconocidos como investigadores, quienes participan en ensayos clínicos en nuestro país deberían haber participado en las etapas que establecimos como requisitos en este estudio, lo que se reconoce como coautoría en las publicaciones generadas.

La persistencia de la concentración de la investigación biomédica en sólo dos universidades, en las últimas tres décadas hace imperioso extenderla a un mayor número de universidades. Esto no sólo para contribuir a generar nuevo conocimiento sino también para familiarizar a los alumnos de pre y post grado con las exigencias de la investiga- ción y para captar vocaciones científicas.

Las características de la investigación biomédica nacional y la relevancia que tiene este aspecto de nuestro desarrollo y cultura nacionales, hacen recomendable que el país realice un esfuerzo para coordinar la investigación en salud, destinarle mayores recursos y centralizar la información temática y financiera de las entidades participantes, estatales y privadas ${ }^{19}$. En cuanto a los ensayos clínicos, deberían cumplir la normativa internacional de estar inscritos, antes de iniciarse, en un Registro oficial con las características dispuestas por la Organización Mundial de la Salud 2,3,20,21.

Habiéndose identificado a los investigadores biomédicos como el grupo mayoritario de investigadores científicos en Chile, es probable que nuestros hallazgos, comentarios y recomendaciones apliquen también para las otras áreas de la investigación científica nacional.

Agradecimientos: A la Sra. Cristina Blamey (secretaria de la Academia Chilena de Medicina) por su colaboración con las encuestas enviadas a los Directores y Encargados de investigación de las Escuelas de Medicina e Institutos de investigación; a la Sra. Silvia Núñez (FONDECYT) por facilitarnos los datos institucionales respectivos; a los Directores y Encargados de investigación de las Universidades: Andrés Bello, Austral de Chile, Católica del Maule, de Chile, del Desarrollo, de Los Andes, de Santiago de Chile, de Valparaíso, Diego Portales, Finis Terra, Mayor, Pontificia Universidad Católica de Chile, y de la Fundación Para la Vida.

\section{Referencias}

1. Global Forum for Health Research. The 10/90 Report on Health Research 2003-2004. Geneva, Switzerland: Global Forum for Health Research, 2004.

2. Alger J, Becerra-Posada F, Kennedy A, Martinelli E, Cuervo LG y Grupo Colaborativo de la Primera Conferencia Latinoamericana de Investigación en Innovación para la Salud. Sistemas nacionales de investigación para la salud en América Latina: una revisión de 14 países. Rev Panam Salud Pública/Pan Am J Public Health 2009; 26: 447-57.

3. Frank C, Nasone E. Health Research: Measuring the social, health and economic benefits. CMAJ 2009; 180: 528-39. 
4. Moloney A. Latin America faces hurdles in health research. Lancet 2009; 374: 1053-4.

5. The Royal Society. The Scientific Century: Securing our future prosperity. RS Policy Document 02/10. March 2010.

6. Fletcher RH, Fletcher SW: Clinical research in general medical journals: A 30-year perspective. N Engl J Med 1979; 301 (4): 180-3.

7. De Angelis C, Drazen JM, Frizelle FA, Haug C, Hoey J, Horton R, Kotzin S, et al. Clinical Trial Registration: A statement from the International Committee of Medical Journal Editors. Ann Intern Med 2004; 141 (6): 477-8.

8. International Committee of Medical Journal Editors: Uniform Requirements for Manuscripts Submitted to Biomedical Journals: Writing and Editing for Biomedical Publication. Updated April 2010. En www.icmje.org

9. Allende J, Babul J, Martínez S, Ureta T. Análisis y proyecciones de la Ciencia Chilena 2005. Academia Chilena de Ciencias, Consejo de Sociedades Científicas, Programa Bicentenario de Ciencias y Tecnología, CONICYT. Alfabeta, Santiago, Chile, 2005.

10. Reyes H. La investigación médica actual en Chile (Editorial). Rev Med Chile 1982; 110: 105-6.

11. Croxatto HR, Vargas-Fernández L, Cruz-Coke R. Estudio sobre la investigación biomédica en el decenio 1967-1976. Encuesta a 49 investigadores de Santiago de Chile. Rev Med Chile 1982; 110: 149-58.

12. Goic A, Armas Merino R, Pérez-Olea J, Reyes H, Cruz-
Coke R, Giraldes E. Situación actual de la investigación en Ciencias Médicas en Chile. Rev Med Chile 1982; 110: 159-73.

13. SCImago. (2007). SJR-SCImago Journal \& Country Rank. Retrieved February 28, 2012, from http://www. scimagojr.com

14. Paraje G. El financiamiento público de la investigación en salud en Chile. Rev Med Chile 2010; 138: 36-43.

15. Romero W, Salas S. ¿Explicitan los autores de la Revista Médica de Chile sus fuentes de financiamiento? Rev Med Chile 2007; 136: 473-9.

16. Federación Latinoamericana de la Industria Farmacéutica. Radiografía de la Investigación Clínica en América Latina. DataFarma-28, Buenos Aires, Argentina, octubre 2010, Año XIV, No 28. Disponible en www.fifarma.org

17. U.S. National Institutes of Health. Clinical Trials.gov. Disponible en www.clinicaltrials.gov

18. Salud al Día. Ediciones Especiales de El Mercurio, 10 de septiembre de 2010.

19. Valdés G, Armas R, Reyes H. Una mirada a la investigación biomédica actual en Chile. Anales de la Academia Chilena de Medicina 2012 (en prensa).

20. Cuervo LG, Valdés A, Clark ML. El registro internacional de ensayos clínicos. Rev Panam Salud Pública 2006; 19 (6): 365-70.

21. Reyes H, Andersen M, Palma J. La Revista Médica de Chile en el año 2009. Rev Med Chile 2009; 137: 108994. 